\title{
Von Neumann's Theory, Projective Measurement, and Quantum Computation
}

\author{
Koji Nagata1, Tadao Nakamura ${ }^{2}$ \\ ${ }^{1}$ Department of Physics, Korea Advanced Institute of Science and Technology, Daejeon, Korea \\ ${ }^{2}$ Department of Information and Computer Science, Keio University, Yokohama, Japan \\ Email: ko mi na@yahoo.co.jp, nakamura@pipelining.jp
}

Received 18 June 2015; accepted 14 July 2015; published 17 July 2015

Copyright (C) 2015 by authors and Scientific Research Publishing Inc.

This work is licensed under the Creative Commons Attribution International License (CC BY). http://creativecommons.org/licenses/by/4.0/

(c) (i) Open Access

\begin{abstract}
We discuss the fact that there is a crucial contradiction within Von Neumann's theory. We derive a proposition concerning a quantum expected value under an assumption of the existence of the orientation of reference frames in $N$ spin-1/2 systems $(1 \leq N<+\infty)$. This assumption intuitively depictures our physical world. However, the quantum predictions within the formalism of Von Neumann's projective measurement violate the proposition with a magnitude that grows exponentially with the number of particles. We have to give up either the existence of the directions or the formalism of Von Neumann's projective measurement. Therefore, Von Neumann's theory cannot depicture our physical world with a violation factor that grows exponentially with the number of particles. The theoretical formalism of the implementation of the Deutsch-Jozsa algorithm relies on Von Neumann's theory. We investigate whether Von Neumann's theory meets the Deutsch-Jozsa algorithm. We discuss the fact that the crucial contradiction makes the quantum-theoretical formulation of Deutsch-Jozsa algorithm questionable. Further, we discuss the fact that projective measurement theory does not meet an easy detector model for a single Pauli observable. Especially, we systematically describe our assertion based on more mathematical analysis using raw data. We propose a solution of the problem. Our solution is equivalent to changing Planck's constant $(\hbar)$ to a new constant $(\hbar / \sqrt{2})$. It may be said that a new type of the quantum theory early approaches Newton's theory in the macroscopic scale than the old quantum theory does. We discuss how our solution is used in an implementation of Deutsch's algorithm.
\end{abstract}

\section{Keywords}

Quantum Computation, Quantum Measurement Theory, Formalism 


\section{Introduction}

Von Neumann introduces the Hilbert space and he tries to present axiomatic system for quantum mechanics [1]. He introduces projective measurement theory. For example, the values of the result of quantum measurements are \pm 1 (in $\hbar / 2$ unit) in spin-1/2 system. An important note here, Von Neumann's theory does not say that we can measure non-commuting observables, simultaneously. Therefore, each value of measurement depends on time even though we do not indicate any suffix concerning time. The detail argument can be seen in Ref. [2] where we indicate a suffix concerning time. Von Neumann's theory is necessary to perform a quantum computer. It is said that there is not any quantum computer without Von Neumann's theory.

A quantum computer is a device for computation that makes direct use of quantum mechanical phenomena, such as superposition and entanglement, to perform operations on data. Quantum computers are different from digital computers based on transistor gates. Whereas digital computers require data to be encoded into binary digits (bits), quantum computation utilizes quantum properties to represent data and perform operations on these data [3]. A theoretical model is the quantum Turing machine, also known as the universal quantum computer. Quantum computers share theoretical similarities with non-deterministic and probabilistic computers, like the ability to be in more than one state simultaneously. The field of quantum computing was first introduced by Richard Feynman in 1982 [4] [5].

As a famous physical theory, the quantum theory (cf. [1] [6]-[10]) gives approximate and at times remarkably accurate numerical predictions. Much experimental data approximately fit to the quantum predictions for the past some 100 years. We do not doubt the correctness of the quantum theory. The quantum theory also says new science with respect to information theory. The science is called the quantum information theory [10]. Therefore, the quantum theory gives us very useful another theory in order to create new information science and to explain the handling of raw experimental data in our physical world.

As for the foundations of the quantum theory, Leggett-type non-local variables theory [11] is experimentally investigated [12]-[14]. The experiments report that the quantum theory does not accept Leggett-type non-local variables interpretation. As for the applications of the quantum theory, implementation of a quantum algorithm to solve Deutsch's problem [15] on a nuclear magnetic resonance quantum computer is reported firstly [16]. Implementation of the Deutsch-Jozsa algorithm on an ion-trap quantum computer is also reported [17]. There are several attempts to use single-photon two-qubit states for quantum computing. Oliveira et al. implement Deutsch's algorithm with polarization and transverse spatial modes of the electromagnetic field as qubits [18]. Single-photon Bell states are prepared and measured [19]. Also the decoherence-free implementation of Deutsch's algorithm is reported by using such single-photon and by using two logical qubits [20]. More recently, a one-way based experimental implementation of Deutsch's algorithm is reported [21].

To date, the quantum theory seems to be a successful physical theory and it looks to have no problems in order to use it experimentally. Several researches address [1] the mathematical formulation of the quantum theory. It is desirable that the quantum theory is also mathematically successful because we predict unknown physical phenomena precisely. Sometimes such predictions are effective in the field of elementary particle physics. We endure much time in order to see the fact by using, for example, a large-scale accelerator. Further, Rolf Landauer says that information is physical [10]. We cannot create any computer without physical phenomena. This fact motivates us to investigate the Hilbert space formalism of the quantum theory. Especially, Von Neumann's theory is accepted widely. Here we ask: Does Von Neumann's theory depicture our physical world? Unfortunately, it is not so even in both the macroscopic scale and the microscopic scale. The theoretical formalism of the implementation of the Deutsch-Jozsa algorithm [15] [22] relies on Von Neumann's theory. Therefore, we cannot implement the Deutsch-Jozsa algorithm by using Von Neumann's theory.

We discuss the fact that there is a crucial contradiction within Von Neumann's theory [2] [23]-[27]. We derive a proposition concerning a quantum expected value under an assumption of the existence of the orientation of reference frames in $N$ spin- $1 / 2$ systems $(1 \leq N<+\infty)$. This assumption intuitively depictures our physical world. However, the quantum predictions within the formalism of Von Neumann's projective measurement "violate" the proposition with a magnitude that grows exponentially with the number of particles. We "have to give up" either the existence of the directions or the formalism of Von Neumann's projective measurement. Therefore, Von Neumann's theory cannot depicture our physical world with a violation factor that grows exponentially with the number of particles. The theoretical formalism of the implementation of the Deutsch-Jozsa algorithm relies on Von Neumann's theory [15] [22]. We reexamine the quantum-theoretical formulation of the 
Deutsch-Jozsa algorithm as the earliest quantum computer. We result in the fact that the formulation is questionable despite the fact that we indeed have raw experimental data.

We know that a theory means a set of propositions. Unfortunately, we have to abandon that the quantum theory satisfies consistency, which is necessary in order to have axiomatic system. This implies that there is no axiomatic system for the quantum theory. A theory $K$ may be said to be consistent if any proposition, $A \in K$, belonging to the theory $K$ and the negation of the proposition, $A\urcorner$, are not derived, simultaneously. Otherwise, the theory $K$ may be said to be contradictory. Our discussion says that, surprisingly, the quantum theory is a contradictory physical theory in order to explain the handling of raw experimental data.

We propose the solution of the problem. Our solution is equivalent to changing Planck's constant $(\hbar)$ to a new constant $(\hbar / \sqrt{2})$. It may be said that a new type of the quantum theory early approaches Newton's theory in the macroscopic scale than the old quantum theory does.

On the other hand, the double-slit experiment is an illustration of wave-particle duality. In it, a beam of particles (such as photons) travels through a barrier with two slits removed. If one puts a detector screen on the other side, the pattern of detected particles shows interference fringes characteristic of waves; however, the detector screen responds to particles. The system exhibits the behaviour of both waves (interference patterns) and particles (dots on the screen).

If we modify this experiment so that one slit is closed, no interference pattern is observed. Thus, the state of both slits affects the final results. We can also arrange to have a minimally invasive detector at one of the slits to detect which slit the particle went through. When we do that, the interference pattern disappears [28]. An analysis of a two-atom double-slit experiment based on environment-induced measurements is reported [29].

We try to implement double-slit experiment. There is a detector just after each slit. Thus interference figure does not appear, and we do not consider such a pattern. The possible values of the result of measurements are \pm 1 (in $\hbar / 2$ unit). If a particle passes one side slit, then the value of the result of measurement is +1 . If a particle passes through another slit, then the value of the result of measurement is -1 . This model is an easy detector model for Pauli observable.

It is discussed [2] [23] [24] that the expected values of two spin observables $\left\langle\sigma_{x}\right\rangle$ and $\left\langle\sigma_{y}\right\rangle$ cannot be measured by using projective measurement theory. And it is discussed that new measurement theory covers the problem. Let us follow the argumentations. Assume a pure spin $1 / 2$ state. We have $\left(\left\langle\sigma_{x}\right\rangle^{2}+\left\langle\sigma_{y}\right\rangle^{2}\right)_{\max }=1$ from the wave functional analysis of quantum mechanics. On the other hand, we have $\left(\left\langle\sigma_{x}\right\rangle^{2}+\left\langle\sigma_{y}\right\rangle^{2}\right)_{\max }=2$ if projective measurement theory is true. Hence the expected values of two spin observables $\left\langle\sigma_{x}\right\rangle$ and $\left\langle\sigma_{y}\right\rangle$ cannot be measured by using projective measurement theory. But, we have $\left(\left\langle\sigma_{x}\right\rangle^{2}+\left\langle\sigma_{y}\right\rangle^{2}\right)_{\max }=1$ when the new quantum measurement theory is true. The different point is that the values of the result of quantum measurements are $\pm 1 / \sqrt{2}$.

It is also discussed [25] [27] that the expected value of a spin observables $\left\langle\sigma_{x}\right\rangle$ cannot be measured by using projective measurement theory. Let us follow the argumentations. Assume a pure spin 1/2 state in the $z$-direction. We have $\left(\left\langle\sigma_{x}\right\rangle^{2}\right)_{\max }=0$ from the wave functional analysis of quantum mechanics. On the other hand, we have $\left(\left\langle\sigma_{x}\right\rangle^{2}\right)_{\max }=1$ if projective measurement theory is true. Hence the expected value of a spin observables $\left\langle\sigma_{x}\right\rangle$ cannot be measured by using projective measurement theory.

We consider whether projective measurement theory meets an easy detector model for Pauli observable. We try to implement double-slit experiment. There is a detector just after each slit. Thus interference figure does not appear, and we do not consider such a pattern. We assume that a source of spin-carrying particles emits them in a state, which can be described as an eigenvector of Pauli observable $\sigma_{z}$. We consider a single expected value of Pauli observable $\sigma_{x}$ in the double-slit experiment. A wave function analysis says that the quantum expected value of it is zero. However, the quantum predictions within projective measurement theory cannot coexist with the value of the expected value of $\left\langle\sigma_{x}\right\rangle=0$. Hence, such projective measurement theory does not meet the easy detector model. We propose a solution of the problem by considering a macroscopic system. We discuss how our solution is used in an implementation of Deutsch's algorithm. Especially, we systematically describe our assertion based on more mathematical analysis using raw data. 
At this stage we are in the following situation.

1) We cannot measure an expected value of a single spin observable by using projective measurement theory.

2) New measurement theory covers the problem mentioned above.

3) We can use new measurement theory for an implementation of Deutsch's algorithm.

Our discussion is very important. The reason is that our discussion reveals that we need new physical theories in order to explain our physical world informationally, to create new information science, and to predict new unknown physical phenomena efficiently. What are new physical theories? We cannot answer it at this stage. However, we expect that our discussion in this paper could contribute to creating new physical theories in order to explain our physical world, to create new information science, and to predict new unknown physical phenomena efficiently.

Throughout this paper, we confine ourselves to the two-level (e.g., electron spin, photon polarizations, and so on) and the discrete eigenvalue case.

Our paper is organized as follows.

In Section 2, we provide the notations and preparation to show a contradiction within Von Neumann's theory.

In Section 3, we discuss the fact that there is a problem within the mathematical formulation of Von Neumann's theory.

In Section 4, we review Deutsch's algorithm along with Ref. [10].

In Section 5, we discuss a problem of Deutsch's algorithm.

In Section 6, we show that Von Neumann's theory does not meet our physical world.

In Section 7, we modify Von Neumann's projective measurement theory.

In Section 8, we propose a new type of the Deutsch-Jozsa algorithm along with our modification of Von Neumann's measurement theory.

In Section 9, we consider the relation between double-slit experiment and projective measurement theory. We cannot measure a single spin observable by using the projective measurement theory.

In Section 10, we consider many double-slit experiments. And we propose a solution of the problem concerning projective measurement theory.

In Section 11, we discuss how our solution is used in an implementation of Deutsch's algorithm.

Section 12 concludes this paper.

\section{Notations and Preparation to Show a Contradiction within Von Neumann's Theory}

We consider a two-dimensional space $H$. Let $\mathbf{N}$ denote a set of the numbers

$$
\{1,2, \cdots,+\infty\}
$$

that contains the countably infinite. Let $S$ be $\{ \pm 1\}$. We assume that every result of measurements lies in $S$. We assume that every time $t$ lies in $\mathbf{N}$. Let $\mathbf{N}_{1}$ denote a set of the numbers

$$
\{1,5,9, \cdots,+\infty\}
$$

that contains the countably infinite. Here we introduce $t_{1} \in \mathbf{N}_{1}$. Let $\mathbf{N}_{2}$ denote a set of the numbers

$$
\{2,6,10, \cdots,+\infty\}
$$

that contains the countably infinite. Here we introduce $t_{2} \in \mathbf{N}_{2}$. Let $\mathbf{N}_{3}$ denote a set of the numbers

$$
\{3,7,11, \cdots,+\infty\}
$$

that contains the countably infinite. Here we introduce $t_{3} \in \mathbf{N}_{3}$. Let $\mathbf{N}_{4}$ denote a set of the numbers

$$
\{4,8,12, \cdots,+\infty\}
$$

that contains the countably infinite. Here we introduce $t_{4} \in \mathbf{N}_{4}$. Let $\sigma$ be

$$
\left(\sigma_{x}, \sigma_{y}, \sigma_{z}\right) \text {, }
$$


the vector of Pauli operators. The measurements (observables) of $\boldsymbol{n} \cdot \boldsymbol{\sigma}$ are parameterized by a unit vector $\boldsymbol{n}$ (its direction along which the spin component is measured). Here, $\cdot$ is the scalar product in $\mathbf{R}^{3}$. One measures an observable $\boldsymbol{n} \cdot \boldsymbol{\sigma}$. We define a notation $\theta(t)$ which represents one result of measurements at time $t$. We assume that measurement of an observable $\boldsymbol{n} \cdot \boldsymbol{\sigma}$ at time $t$ for a physical system in a state $\psi$ yields a value $\theta(\psi, \boldsymbol{n} \cdot \boldsymbol{\sigma}, t) \in S$.

We consider the following propositions:

Proposition: $M$ (measurement outcome),

$$
\theta(\psi, \boldsymbol{n} \cdot \boldsymbol{\sigma}, t) \in S
$$

Proposition: E (quantum expected value),

$$
\operatorname{Tr}[\psi \boldsymbol{n} \cdot \boldsymbol{\sigma}]=\lim _{m \rightarrow \infty} \frac{\sum_{t=1}^{m} \theta(\psi, \boldsymbol{n} \cdot \boldsymbol{\sigma}, t)}{m} .
$$

Lemma: $T$

if

$$
\operatorname{Tr}[\psi \boldsymbol{n} \cdot \boldsymbol{\sigma}]=\lim _{m \rightarrow \infty} \frac{\sum_{t=1}^{m} \theta(\psi, \boldsymbol{n} \cdot \boldsymbol{\sigma}, t)}{m},
$$

then

$$
\operatorname{Tr}[\psi \boldsymbol{n} \cdot \boldsymbol{\sigma}]=\lim _{m_{1} \rightarrow \infty} \frac{\sum_{t_{1}=1}^{m_{1}} \theta\left(\psi, \boldsymbol{n} \cdot \boldsymbol{\sigma}, t_{1}\right)}{m_{1}}=\lim _{m_{2} \rightarrow \infty} \frac{\sum_{t_{2}=2}^{m_{2}} \theta\left(\psi, \boldsymbol{n} \cdot \boldsymbol{\sigma}, t_{2}\right)}{m_{2}}
$$

and

$$
\operatorname{Tr}[\psi \boldsymbol{n} \cdot \boldsymbol{\sigma}]=\lim _{m_{3} \rightarrow \infty} \frac{\sum_{t_{3}=3}^{m_{3}} \theta\left(\psi, \boldsymbol{n} \cdot \boldsymbol{\sigma}, t_{3}\right)}{m_{3}}=\lim _{m_{4} \rightarrow \infty} \frac{\sum_{t_{4}=4}^{m_{4}} \theta\left(\psi, \boldsymbol{n} \cdot \boldsymbol{\sigma}, t_{4}\right)}{m_{4}} .
$$

\section{Whether Von Neumann's Theory Can Be Almighty}

In this section, we investigate if Von Neumann’s theory can be almighty.

\subsection{The Existence of the Orientation of Reference Frames}

We assume a pure spin- $1 / 2$ state $\psi$ lying in the $x-y$ plane. Let $\sigma$ be $\left(\sigma_{x}, \sigma_{y}, \sigma_{z}\right)$, the vector of Pauli operators. The measurements (observables) on a spin-1/2 state lying in the $x$-y plane of $\boldsymbol{n} \cdot \boldsymbol{\sigma}$ are parameterized by a unit vector $\boldsymbol{n}$ (its direction along which the spin component is measured). Here, $\cdot$ is the scalar product in $\mathbf{R}^{3}$.

We have a quantum expected value $E_{\mathrm{QM}}^{k}, k=1,2$ as

$$
E_{\mathrm{QM}}^{k} \equiv \operatorname{Tr}\left[\psi \boldsymbol{n}_{k} \cdot \boldsymbol{\sigma}\right], k=1,2 .
$$

We have $\boldsymbol{x} \equiv \boldsymbol{x}^{(1)}, \boldsymbol{y} \equiv \boldsymbol{x}^{(2)}$, and $\mathbf{z} \equiv \boldsymbol{x}^{(3)}$. They are the Cartesian axes relative to which spherical angles are measured. We write two unit vectors in the plane defined by $\boldsymbol{x}^{(1)}$ and $\boldsymbol{x}^{(2)}$ in the following way:

$$
\boldsymbol{n}_{k}=\cos \theta_{k} \boldsymbol{x}^{(1)}+\sin \theta_{k} \boldsymbol{x}^{(2)} .
$$

Here, the angle $\theta_{k}$ takes only two values:

$$
\theta_{1}=0, \theta_{2}=\frac{\pi}{2}
$$


We derive a necessary condition for the quantum expected value for the system in a pure spin- $1 / 2$ state lying in the $x-y$ plane given in (3.1). We derive the possible values of the scalar product

$$
\sum_{k=1}^{2}\left(E_{\mathrm{QM}}^{k} \times E_{\mathrm{QM}}^{k}\right) \equiv\left\|E_{\mathrm{QM}}\right\|^{2} \text {. }
$$

$E_{\mathrm{QM}}^{k}$ is the quantum expected value given in (3.1). We see that

$$
\left\|E_{\mathrm{QM}}\right\|^{2}=\left\langle\sigma_{x}\right\rangle^{2}+\left\langle\sigma_{y}\right\rangle^{2} .
$$

We use the decomposition (3.2). We introduce simplified notations as

$$
T_{i}=\operatorname{Tr}\left[\psi \boldsymbol{x}^{(i)} \cdot \boldsymbol{\sigma}\right]
$$

and

$$
\left(c_{k}^{1}, c_{k}^{2}\right)=\left(\cos \theta_{k}, \sin \theta_{k}\right)
$$

Then, we have

$$
\left\|E_{\mathrm{QM}}\right\|^{2}=\sum_{k=1}^{2}\left(\sum_{i=1}^{2} T_{i} C_{k}^{i}\right)^{2}=\sum_{i=1}^{2} T_{i}^{2} \leq 1
$$

where we use the orthogonality relation

$$
\sum_{k=1}^{2} c_{k}^{\alpha} c_{k}^{\beta}=\delta_{\alpha, \beta}
$$

From a proposition of the quantum theory, the Bloch sphere (the orientation of reference frames) with the value of

$$
\sum_{i=1}^{2} T_{i}^{2}
$$

is bounded as

$$
\sum_{i=1}^{2} T_{i}^{2} \leq 1
$$

The reason of the condition (3.8) is the Bloch sphere

$$
\sum_{i=1}^{3}\left(\operatorname{Tr}\left[\psi \boldsymbol{x}^{(i)} \cdot \boldsymbol{\sigma}\right]\right)^{2} \leq 1
$$

Thus we derive a proposition concerning a quantum expected value under an assumption of the existence of the orientation of reference frames (in a spin-1/2 system). The proposition is

$$
\left\|E_{\mathrm{QM}}\right\|^{2} \leq 1 \text {. }
$$

This inequality is saturated and iff $\psi$ is a pure state lying in the $x-y$ plane. That is,

$$
\sum_{i=1}^{2}\left(\operatorname{Tr}\left[\psi \boldsymbol{x}^{(i)} \cdot \boldsymbol{\sigma}\right]\right)^{2}=1
$$

Hence, we derive the following proposition concerning the existence of the orientation of reference frames when the system is in a pure state lying in the $x$-y plane

$$
\left\|E_{\mathrm{QM}}\right\|_{\max }^{2}=1 .
$$




\subsection{The Existence of Measurement Outcome}

We assign the truth value "1" for Proposition $M$ and Proposition $E$. Let $A_{k}$ be $\boldsymbol{n}_{k} \cdot \boldsymbol{\sigma}$. We assume four gedanken experiments in the same state $\psi$. The value of $\theta\left(\psi, A_{1}, t_{1}\right)$ is independent of $\theta\left(\psi, A_{1}, t_{2}\right)$. We note that the measurement time is different from each other. Here, we assume $t_{1} \in \mathbf{N}_{1}$ and $t_{2} \in \mathbf{N}_{2}$. The value of $\theta\left(\psi, A_{2}, t_{3}\right)$ is independent of $\theta\left(\psi, A_{2}, t_{4}\right)$. We note that the measurement time is different from each other. Here, we assume $t_{3} \in \mathbf{N}_{3}$ and $t_{4} \in \mathbf{N}_{4}$. The values of $\theta\left(\psi, A_{1}, t_{1}\right), \theta\left(\psi, A_{1}, t_{2}\right), \theta\left(\psi, A_{2}, t_{3}\right)$, and $\theta\left(\psi, A_{2}, t_{4}\right)$ are independent of each other. We note that the measurement time is different from each other. We assume that the number of each of quantum measurements is the countably infinite. We know that a sum of "four" countably infinite is the countably infinite. We do not have to assign definite values to non-commuting observables in the same time.

From Proposition $E$ and Lemma $T$, the quantum expected value in (3.1) $(k=1)$, which is the average of the results of measurements, is given by

$$
E_{\mathrm{QM}}^{1}=\lim _{m_{1} \rightarrow \infty} \frac{\sum_{t_{1}=1}^{m_{1}} \theta\left(\psi, A_{1}, t_{1}\right)}{m_{1}} .
$$

From Proposition $M$, the possible values of the actually measured result $\theta\left(\psi, A_{1}, t_{1}\right)$ are \pm 1 .

From Lemma $T$, the same quantum expected value is given by

$$
E_{\mathrm{QM}}^{1}=\lim _{m_{2} \rightarrow \infty} \frac{\sum_{t_{2}=2}^{m_{2}} \theta\left(\psi, A_{1}, t_{2}\right)}{m_{2}} .
$$

From Proposition $M$, the possible values of the actually measured result $\theta\left(\psi, A_{1}, t_{2}\right)$ are \pm 1 . From Lemma $T$, we see

$$
\begin{aligned}
& \left\|\left\{t_{1} \mid t_{1} \in \mathbf{N}_{1} \wedge \theta\left(\psi, A_{1}, t_{1}\right)=1\right\}\right\|=\left\|\left\{t_{2} \mid t_{2} \in \mathbf{N}_{2} \wedge \theta\left(\psi, A_{1}, t_{2}\right)=1\right\}\right\|, \\
& \left\|\left\{t_{1} \mid t_{1} \in \mathbf{N}_{1} \wedge \theta\left(\psi, A_{1}, t_{1}\right)=-1\right\}\right\|=\left\|\left\{t_{2} \mid t_{2} \in \mathbf{N}_{2} \wedge \theta\left(\psi, A_{1}, t_{2}\right)=-1\right\}\right\| .
\end{aligned}
$$

From Proposition $E$ and Lemma $T$, the quantum expected value in (3.1) $(k=2)$, which is the average of the results of measurements, is given by

$$
E_{\mathrm{QM}}^{2}=\lim _{m_{3} \rightarrow \infty} \frac{\sum_{t_{3}=3}^{m_{3}} \theta\left(\psi, A_{2}, t_{3}\right)}{m_{3}} .
$$

From Proposition $M$, the possible values of the actually measured result $\theta\left(\psi, A_{2}, t_{3}\right)$ are \pm 1 .

From Lemma $T$, the same quantum expected value is given by

$$
E_{\mathrm{QM}}^{2}=\lim _{m_{4} \rightarrow \infty} \frac{\sum_{t_{4}=4}^{m_{4}} \theta\left(\psi, A_{2}, t_{4}\right)}{m_{4}} .
$$

From Proposition $M$, the possible values of the actually measured result $\theta\left(\psi, A_{2}, t_{4}\right)$ are \pm 1 . From Lemma $T$, we see

$$
\begin{aligned}
& \left\|\left\{t_{3} \mid t_{3} \in \mathbf{N}_{3} \wedge \theta\left(\psi, A_{2}, t_{3}\right)=1\right\}\right\|=\left\|\left\{t_{4} \mid t_{4} \in \mathbf{N}_{4} \wedge \theta\left(\psi, A_{2}, t_{4}\right)=1\right\}\right\|, \\
& \left\|\left\{t_{3} \mid t_{3} \in \mathbf{N}_{3} \wedge \theta\left(\psi, A_{2}, t_{3}\right)=-1\right\}\right\|=\left\|\left\{t_{4} \mid t_{4} \in \mathbf{N}_{4} \wedge \theta\left(\psi, A_{2}, t_{4}\right)=-1\right\}\right\| .
\end{aligned}
$$

We derive a necessary condition for the two quantum expected values for the system in a pure spin- $1 / 2$ state lying in the $x-y$ plane given in (3.16) and (3.19). We derive the possible values of the scalar product $\left\|E_{\mathrm{QM}}\right\|^{2}$ of the two quantum expected values, $E_{\mathrm{QM}}^{k}$ given in (3.16) and (3.19). We have 


$$
\begin{aligned}
\left\|E_{\mathrm{QM}}\right\|^{2} & =\left(\lim _{m_{1} \rightarrow \infty} \frac{\sum_{t_{1}=1}^{m_{1}} \theta\left(\psi, A_{1}, t_{1}\right)}{m_{1}} \times \lim _{m_{2} \rightarrow \infty} \frac{\sum_{t_{2}=2}^{m_{2}} \theta\left(\psi, A_{1}, t_{2}\right)}{m_{2}}\right)+\left(\lim _{m_{3} \rightarrow \infty} \frac{\sum_{t_{3}=3}^{m_{3}} \theta\left(\psi, A_{2}, t_{3}\right)}{m_{3}} \times \lim _{m_{4} \rightarrow \infty} \frac{\sum_{t_{4}=4}^{m_{4}} \theta\left(\psi, A_{2}, t_{4}\right)}{m_{4}}\right) \\
& =\left(\lim _{m_{1} \rightarrow \infty} \frac{\sum_{t_{1}=1}^{m_{1}}}{m_{1}} \cdot \lim _{m_{2} \rightarrow \infty} \frac{\sum_{t_{2}=2}^{m_{2}}}{m_{2}} \theta\left(\psi, A_{1}, t_{1}\right) \theta\left(\psi, A_{1}, t_{2}\right)\right)+\left(\lim _{m_{3} \rightarrow \infty} \frac{\sum_{t_{3}=3}^{m_{3}}}{m_{3}} \cdot \lim _{m_{4} \rightarrow \infty} \frac{\sum_{t_{4}=4}^{m_{4}}}{m_{4}} \theta\left(\psi, A_{2}, t_{3}\right) \theta\left(\psi, A_{2}, t_{4}\right)\right) \\
& \leq\left(\lim _{m_{1} \rightarrow \infty} \frac{\sum_{t_{1}=1}^{m_{1}}}{m_{1}} \cdot \lim _{m_{2} \rightarrow \infty} \frac{\sum_{t_{2}=2}^{m_{2}}}{m_{2}}\left|\theta\left(\psi, A_{1}, t_{1}\right) \theta\left(\psi, A_{1}, t_{2}\right)\right|+\left(\sum_{m_{3} \rightarrow \infty} \frac{\sum_{t_{3}=3}^{m_{3}}}{m_{3}} \cdot \lim _{m_{4} \rightarrow \infty} \frac{\sum_{t_{4}=4}^{m_{4}}}{m_{4}} \mid \theta\left(\psi, A_{2}, t_{3}\right) \theta\left(\psi, A_{2}, t_{4}\right)\right)\right. \\
& =\left(\sum_{m_{1} \rightarrow \infty} \frac{\sum_{t_{1}=1}^{m_{1}}}{m_{1}} \cdot \lim _{m_{2} \rightarrow \infty} \frac{\sum_{t_{2}=2}^{m_{2}}}{m_{2}}\right)+\left(\sum_{m_{3} \rightarrow \infty} \frac{\sum_{t_{3}=3}^{m_{3}} \cdot \sum_{m_{3}}^{m_{3}} \sum_{m_{4} \rightarrow \infty}}{m_{4}=4}\right)=2 .
\end{aligned}
$$

From Proposition $M$, we have

$$
\left|\theta\left(\psi, A_{1}, t_{1}\right) \theta\left(\psi, A_{1}, t_{2}\right)\right|=+1,\left|\theta\left(\psi, A_{2}, t_{3}\right) \theta\left(\psi, A_{2}, t_{4}\right)\right|=+1 .
$$

The above inequality (3.22) is saturated when

$$
\theta\left(\psi, A_{1}, t_{1}\right) \theta\left(\psi, A_{1}, t_{2}\right)=1, \theta\left(\psi, A_{2}, t_{3}\right) \theta\left(\psi, A_{2}, t_{4}\right)=1 .
$$

This implies

$$
\theta\left(\psi, A_{1}, t_{1}\right)=\theta\left(\psi, A_{1}, t_{2}\right), \theta\left(\psi, A_{2}, t_{3}\right)=\theta\left(\psi, A_{2}, t_{4}\right) .
$$

The above condition (3.25) can be possible since, as we have said,

$$
\begin{aligned}
& \left\|\left\{t_{1} \mid t_{1} \in \mathbf{N}_{1} \wedge \theta\left(\psi, A_{1}, t_{1}\right)=1\right\}\right\|=\left\|\left\{t_{2} \mid t_{2} \in \mathbf{N}_{2} \wedge \theta\left(\psi, A_{1}, t_{2}\right)=1\right\}\right\|, \\
& \left\|\left\{t_{1} \mid t_{1} \in \mathbf{N}_{1} \wedge \theta\left(\psi, A_{1}, t_{1}\right)=-1\right\}\right\|=\left\|\left\{t_{2} \mid t_{2} \in \mathbf{N}_{2} \wedge \theta\left(\psi, A_{1}, t_{2}\right)=-1\right\}\right\| .
\end{aligned}
$$

and

$$
\begin{aligned}
& \left\|\left\{t_{3} \mid t_{3} \in \mathbf{N}_{3} \wedge \theta\left(\psi, A_{2}, t_{3}\right)=1\right\}\right\|=\left\|\left\{t_{4} \mid t_{4} \in \mathbf{N}_{4} \wedge \theta\left(\psi, A_{2}, t_{4}\right)=1\right\}\right\|, \\
& \left\|\left\{t_{3} \mid t_{3} \in \mathbf{N}_{3} \wedge \theta\left(\psi, A_{2}, t_{3}\right)=-1\right\}\right\|=\left\|\left\{t_{4} \mid t_{4} \in \mathbf{N}_{4} \wedge \theta\left(\psi, A_{2}, t_{4}\right)=-1\right\}\right\| .
\end{aligned}
$$

Thus we derive a proposition concerning the two quantum expected values under an assumption that we assign the truth value " 1 ” for Proposition $M$ and Proposition $E$, (in a spin-1/2 system). The proposition is $\left\|E_{\mathrm{QM}}\right\|^{2} \leq 2$. This inequality can be saturated. Hence we derive the following proposition concerning Proposition $M$ and Proposition E:

$$
\left\|E_{\mathrm{QM}}\right\|_{\max }^{2}=2 .
$$

We cannot assign the truth value " 1 " for two propositions (3.15) (concerning the existence of the orientation of reference frames) and (3.28) (concerning Proposition $M$ and Proposition $E$ ), simultaneously, when the system is in a pure state lying in the $x-y$ plane. We do not assign the truth value " 1 " for three propositions:

1) Proposition $M$; 
2) Proposition E;

3) The existence of the orientation of reference frames simultaneously. In other words, we do not assign the truth value " 1 " for two propositions:

a) The existence of measurement outcome;

b) The existence of the orientation of reference frames simultaneously.

\section{Quantum Computation}

In this section, we review Deutsch's algorithm along with Ref. [10].

Quantum parallelism is a fundamental feature of many quantum algorithms. It allows quantum computers to evaluate the values of a function $f(x)$ for many different values of $x$ simultaneously. Suppose

$$
f:\{0,1\} \rightarrow\{0,1\}
$$

is a function with a one-bit domain and range. A convenient way of computing this function on a quantum computer is to consider a two-qubit quantum computer which starts in the state

$$
|x, y\rangle \text {. }
$$

With an appropriate sequence of logic gates it is possible to transform this state into

$$
|x, y \oplus f(x)\rangle,
$$

where $\oplus$ indicates addition modulo 2 . We give the transformation defined by the map

$$
|x, y\rangle \rightarrow|x, y \oplus f(x)\rangle
$$

a name, $U_{f}$.

Deutsch's algorithm combines quantum parallelism with a property of quantum mechanics known as interference. Let us use the Hadamard gate to prepare the first qubit

as the superposition

$$
(|0\rangle+|1\rangle) / \sqrt{2},
$$

but let us prepare the second qubit as the superposition

$$
(|0\rangle-|1\rangle) / \sqrt{2},
$$

using the Hadamard gate applied to the state

$$
|1\rangle \text {. }
$$

The Hadamard gate is as

$$
H=\frac{1}{\sqrt{2}}(|0\rangle\langle 1|+| 1\rangle\langle 0|+| 0\rangle\langle 0|-| 1\rangle\langle 1|) .
$$

Let us follow the states along to see what happens in this circuit. The input state

$$
\left|\psi_{0}\right\rangle=|01\rangle
$$

is sent through two Hadamard gates to give

$$
\left|\psi_{1}\right\rangle=\left[\frac{|0\rangle+|1\rangle}{\sqrt{2}}\right]\left[\frac{|0\rangle-|1\rangle}{\sqrt{2}}\right] .
$$

A little thought shows that if we apply $U_{f}$ to the state 


$$
|x\rangle(|0\rangle-|1\rangle) / \sqrt{2}
$$

then we obtain the state

$$
(-1)^{f(x)}|x\rangle(|0\rangle-|1\rangle) / \sqrt{2}
$$

Applying $U_{f}$ to $\left|\psi_{1}\right\rangle$ therefore leaves us with one of the two possibilities:

$$
\left|\psi_{2}\right\rangle=\left\{\begin{array}{l} 
\pm\left[\frac{|0\rangle+|1\rangle}{\sqrt{2}}\right]\left[\frac{|0\rangle-|1\rangle}{\sqrt{2}}\right] \quad \text { if } f(0)=f(1) \\
\pm\left[\frac{|0\rangle-|1\rangle}{\sqrt{2}}\right]\left[\frac{|0\rangle-|1\rangle}{\sqrt{2}}\right] \text { if } f(0) \neq f(1) .
\end{array}\right.
$$

The final Hadamard gate on the first qubit thus gives us

$$
\left|\psi_{3}\right\rangle= \begin{cases} \pm|0\rangle\left[\frac{|0\rangle-|1\rangle}{\sqrt{2}}\right] & \text { if } f(0)=f(1) \\ \pm|1\rangle\left[\frac{|0\rangle-|1\rangle}{\sqrt{2}}\right] & \text { if } f(0) \neq f(1) .\end{cases}
$$

Realizing that $f(0) \oplus f(1)$ is 0 if $f(0)=f(1)$ and 1 otherwise, we can rewrite this result concisely as

$$
\left|\psi_{3}\right\rangle= \pm|f(0) \oplus f(1)\rangle\left[\frac{|0\rangle-|1\rangle}{\sqrt{2}}\right],
$$

so by measuring the first qubit we may determine $f(0) \oplus f(1)$. This is very interesting indeed: the quantum circuit gives us the ability to determine a global property of $f(x)$, namely $f(0) \oplus f(1)$, using only one evaluation of $f(x)$ ! This is faster than is possible with a classical apparatus, which would require at least two evaluations.

\section{Problem of Deutsch's Algorithm}

In this section, we suggest a problem of Deutsch's algorithm. We see that the implementation of Deutsch's algorithm is not possible if we give up either observability of a quantum state or controllability of a quantum state.

We introduce the following quantum proposition concerning controllability:

$$
\langle 0 \mid 0\rangle=1,\langle 1 \mid 1\rangle=1,\langle 0 \mid 1\rangle=0 \text {, and }\langle 1 \mid 0\rangle=0 .
$$

We may consider the following non-quantum-theoretical proposition:

$$
\langle 0 \mid 0\rangle=-1,\langle 1 \mid 1\rangle=-1,\langle 0 \mid 1\rangle=0 \text {, and }\langle 1 \mid 0\rangle=0 .
$$

The proposition (5.2) implies the validity of Proposition $M$ and Proposition $E$ (observability and the existence of measurement outcome). The proposition (5.2) implies

$$
|\langle 0 \mid 0\rangle|^{2}=1,|\langle 1 \mid 1\rangle|^{2}=1,|\langle 0 \mid 1\rangle|^{2}=0 \text {, and }|\langle 1 \mid 0\rangle|^{2}=0 .
$$

Thus,

$$
\begin{aligned}
& \operatorname{Tr}[|0\rangle\langle 0|| 0\rangle\langle 0|]=1, \operatorname{Tr}[|1\rangle\langle 1|| 1\rangle\langle 1|]=1, \\
& \operatorname{Tr}[|0\rangle\langle 0|| 1\rangle\langle 1|]=0, \text { and } \operatorname{Tr}[|1\rangle\langle 1|| 0\rangle\langle 0|]=0 .
\end{aligned}
$$

However, the validity of Proposition $M$ and Proposition $E$ does not imply the proposition (5.2). We see that the 
proposition (5.1) is not equivalent to Proposition $M$ and Proposition $E$ (observability and the existence of measurement outcome). From Truth Value Table 1, we can assign the truth value "1" for the proposition (5.2), Proposition $M$, and Proposition $E$ (observability and the existence of measurement outcome) and we can assign the truth value " 0 " for the proposition (5.1) concerning controllability.

On the other hand, the proposition (5.1) implies that

$$
\left\|E_{\mathrm{QM}}\right\|^{2}=\left\langle\sigma_{x}\right\rangle^{2}+\left\langle\sigma_{y}\right\rangle^{2}=1
$$

when the system is in a pure state lying in the $x-y$ plane. The reason is as follows: Assume a pure state lying in the $x-y$ plane as

$$
|\psi\rangle=\frac{|0\rangle\rangle+\mathrm{e}^{i \phi}|1\rangle}{\sqrt{2}}
$$

where $\phi$ is a phase. Let us write

$$
\sigma_{x}=|0\rangle\langle 1|+| 1\rangle\langle 0|
$$

and

$$
\sigma_{y}=-i|0\rangle\langle 1|+i| 1\rangle\langle 0|
$$

Then we have

$$
\left\langle\psi\left|\sigma_{x}\right| \psi\right\rangle=\cos (\phi)
$$

and

$$
\left\langle\psi\left|\sigma_{y}\right| \psi\right\rangle=\sin (\phi) .
$$

Therefore, we see

$$
\left\langle\psi\left|\sigma_{x}\right| \psi\right\rangle^{2}+\left\langle\psi\left|\sigma_{y}\right| \psi\right\rangle^{2}=\cos ^{2}(\phi)+\sin ^{2}(\phi)=1 .
$$

We thus see the proposition (5.1) implies the existence of the orientation of reference frames in the Hilbert space formalism of the quantum theory.

From the discussion presented in Section 3, we see that the quantum proposition (5.1) concerning controllability (the existence of the orientation of reference frames) cannot coexist with the validity of Proposition $M$ and Proposition $E$ (observability and the existence of measurement outcome), which states

$$
\left\|E_{\mathrm{QM}}\right\|_{\max }^{2}=2 \text {, }
$$

when the system is in a pure state lying in the $x-y$ plane.

Table 1. Truth value table: $A$ implies a proposition (5.1) concerning controllability. $B$ implies a non-quantum-theoretical proposition (5.2). $A \vee B$ implies a proposition (5.3) of disjunction of $A$ and $B$ concerning observability.

\begin{tabular}{ccc}
$A$ & $B$ & $A \vee B$ \\
\hline 1 & 1 & 1 \\
1 & 0 & 1 \\
0 & 1 & 1 \\
0 & 0 & 0
\end{tabular}


Deutsch's algorithm shows the importance of the ability of the Hadamard gate (controllability and the existence of the orientation of reference frames) for quantum computation. The ability of the Hadamard gate is valid only when we assign the truth value " 1 " for the proposition (5.1) (the existence of the orientation of reference frames). We see that the quantum state

$$
(|0\rangle \pm|1\rangle) / \sqrt{2}
$$

is a pure state lying in the $x-y$ plane. We can assign the truth value " 1 " for the ability of the Hadamard gate (controllability and the existence of the orientation of reference frames)

$$
H\left(\frac{|0\rangle+|1\rangle}{\sqrt{2}}\right)=|0\rangle, \quad H\left(\frac{|0\rangle-|1\rangle}{\sqrt{2}}\right)=|1\rangle
$$

only when we assign the truth value " 1 " for the proposition (5.1) concerning controllability (the existence of the orientation of reference frames) and we give up the validity of Proposition $M$ and Proposition $E$ (observability and the existence of measurement outcome). The validity of the proposition (5.1) implies that

$$
H^{2}=I \text {. }
$$

Thus applying $H$ twice to a quantum state does nothing to it if we assign the truth value "1" for the proposition (5.1). When we assign the truth value "1" for the proposition (5.1), we have

$$
\frac{|0\rangle+|1\rangle}{\sqrt{2}}=H|0\rangle, \frac{|0\rangle-|1\rangle}{\sqrt{2}}=H|1\rangle .
$$

We conclude that the step in which transforms the state $\left|\psi_{0}\right\rangle$ into the state $\left|\psi_{1}\right\rangle$, namely the step saying from (4.10) to (4.11) is possible only when we assign the truth value " 1 " for the proposition (5.1) (concerning controllability and the existence of the orientation of reference frames) and we give up the validity of Proposition $M$ and Proposition $E$ (concerning observability and the existence of measurement outcome). The step saying from (4.14) to (4.15) is also so. Therefore we question what makes observability if we assign the truth value "1" for the ability of the Hadamard gate (controllability and the existence of the orientation of reference frames). We also question what makes controllability if we assign the truth value " 1 " for Proposition $M$ and Proposition $E$ (observability and the existence of measurement outcome).

\section{Von Neumann's Theory Does Not Meet Our Physical World}

Assume that we have a set of $N$ spins $\frac{1}{2}$. Each of them is a spin-1/2 pure state lying in the $x-y$ plane. Let us assume that one source of $N$ uncorrelated spin-carrying particles emits them in a state, which can be described as a multi spin-1/2 pure uncorrelated state. Let us parameterize the settings of the jth observer with a unit vector $\boldsymbol{n}_{j}$ (its direction along which the spin component is measured) with $j=1, \cdots, N$. One can introduce the "projective" correlation function, which is the average of the product of the results of Von Neumann's projective measurement

$$
E_{\mathrm{PM}}\left(\boldsymbol{n}_{1}, \boldsymbol{n}_{2}, \cdots, \boldsymbol{n}_{N}\right)=\left\langle r\left(\boldsymbol{n}_{1}, \boldsymbol{n}_{2}, \cdots, \boldsymbol{n}_{N}\right)\right\rangle_{\text {avg }},
$$

where $r$ is the projective result. We assume the value of $r$ is \pm 1 (in $(\hbar / 2)^{N}$ unit), which is obtained if the measurement directions are set at $\boldsymbol{n}_{1}, \boldsymbol{n}_{2}, \cdots, \boldsymbol{n}_{N}$.

Also one can introduce a quantum correlation function with the system in such a pure uncorrelated state

$$
E_{\mathrm{QM}}\left(\boldsymbol{n}_{1}, \boldsymbol{n}_{2}, \cdots, \boldsymbol{n}_{N}\right)=\operatorname{tr}\left[\rho \boldsymbol{n}_{1} \cdot \boldsymbol{\sigma} \otimes \boldsymbol{n}_{2} \cdot \boldsymbol{\sigma} \otimes \cdots \otimes \boldsymbol{n}_{N} \cdot \boldsymbol{\sigma}\right]
$$

where $\otimes$ denotes the tensor product, - the scalar product in $\mathbf{R}^{2}, \sigma=\left(\sigma_{x}, \sigma_{y}\right)$ is a vector of two Pauli operators, and $\rho$ is the pure uncorrelated state,

$$
\rho=\rho_{1} \otimes \rho_{2} \otimes \cdots \otimes \rho_{N}
$$


with $\rho_{j}=\left|\Psi_{j}\right\rangle\left\langle\Psi_{j}\right|$ and $\left|\Psi_{j}\right\rangle$ is a spin-1/2 pure state lying in the $x-y$ plane.

One can write the observable (unit) vector $\boldsymbol{n}_{j}$ in a plane coordinate system as follows:

$$
\boldsymbol{n}_{j}\left(\theta_{j}^{k_{j}}\right)=\cos \theta_{j}^{k_{j}} \boldsymbol{x}_{j}^{(1)}+\sin \theta_{j}^{k_{j}} \boldsymbol{x}_{j}^{(2)},
$$

where $\boldsymbol{x}_{j}^{(1)}=\boldsymbol{x}$ and $\boldsymbol{x}_{j}^{(2)}=\boldsymbol{y}$ are the Cartesian axes. Here, the angle $\theta_{j}^{k_{j}}$ takes two values (two-setting model):

$$
\theta_{j}^{1}=0, \theta_{j}^{2}=\frac{\pi}{2}
$$

We derive a necessary condition to be satisfied by the quantum correlation function with the system in a pure uncorrelated state given in (6.2). In more detail, we derive the value of the product of the quantum correlation function, $E_{\mathrm{QM}}$ given in (6.2), i.e., $\left\|E_{\mathrm{QM}}\right\|^{2}$. We use the decomposition (6.4). We introduce simplified notations as

$$
T_{i_{1} i_{2} \cdots i_{N}}=\operatorname{tr}\left[\rho \boldsymbol{x}_{1}^{\left(i_{1}\right)} \cdot \boldsymbol{\sigma} \otimes \boldsymbol{x}_{2}^{\left(i_{2}\right)} \cdot \boldsymbol{\sigma} \otimes \cdots \otimes \boldsymbol{x}_{N}^{\left(i_{N}\right)} \cdot \boldsymbol{\sigma}\right]
$$

and

$$
\boldsymbol{c}_{j}=\left(c_{j}^{1}, c_{j}^{2}\right)=\left(\cos \theta_{j}^{k_{j}}, \sin \theta_{j}^{k_{j}}\right)
$$

Then, we have

$$
\left\|E_{\mathrm{QM}}\right\|^{2}=\sum_{k_{1}=1}^{2} \cdots \sum_{k_{N}=1}^{2}\left(\sum_{i_{1}, \cdots, i_{N}=1}^{2} T_{i_{1} \cdots i_{N}} c_{1}^{i_{1}} \cdots c_{N}^{i_{N}}\right)^{2}=\sum_{i_{1}, \cdots, i_{N}=1}^{2} T_{i_{1} \cdots i_{N}}^{2} \leq 1,
$$

where we use the orthogonality relation $\sum_{k_{j}=1}^{2} c_{j}^{\alpha} c_{j}^{\beta}=\delta_{\alpha, \beta}$. The value of $\sum_{i_{1}, \cdots, i_{N}=1}^{2} T_{i_{1} \cdots i_{N}}^{2}$ is bounded as $\sum_{i_{1}, \cdots, i_{N}=1}^{2} T_{i_{1} \cdots i_{N}}^{2} \leq 1$. We have

$$
\prod_{j=1 i_{j}=1}^{N} \sum^{2}\left(\operatorname{tr}\left[\rho_{j} x_{j}^{\left(i_{j}\right)} \cdot \boldsymbol{\sigma}\right]\right)^{2} \leq 1
$$

From the convex argument, all quantum separable states must satisfy the inequality (6.8). Therefore, it is a separability inequality. It is important that the separability inequality (6.8) is saturated iff $\rho$ is a multi spin-1/2 pure uncorrelated state such that, for every $j,\left|\Psi_{j}\right\rangle$ is a spin-1/2 pure state lying in the $x$-y plane. The reason of the inequality (6.8) is due to the following quantum inequality

$$
\sum_{i_{j}=1}^{2}\left(\operatorname{tr}\left[\rho_{j} \boldsymbol{x}_{j}^{\left(i_{j}\right)} \cdot \boldsymbol{\sigma}\right]\right)^{2} \leq 1 .
$$

The inequality (6.10) is saturated iff $\rho_{j}=\left|\Psi_{j}\right\rangle\left\langle\Psi_{j}\right|$ and $\left|\Psi_{j}\right\rangle$ is a spin-1/2 pure state lying in the $x-y$ plane. The inequality (6.8) is saturated iff the inequality $(6.10)$ is saturated for every $j$. Thus we have the maximal possible value of the scalar product as a quantum proposition concerning our physical world

$$
\left\|E_{\mathrm{QM}}\right\|_{\max }^{2}=1
$$

when the system is in such a multi spin-1/2 pure uncorrelated state.

On the other hand, a correlation function satisfies projective measurement theory if it can be written as

$$
E_{\mathrm{PM}}\left(\boldsymbol{n}_{1}, \boldsymbol{n}_{2}, \cdots, \boldsymbol{n}_{N}\right)=\lim _{m \rightarrow \infty} \frac{\sum_{l=1}^{m} r\left(\boldsymbol{n}_{1}, \boldsymbol{n}_{2}, \cdots, \boldsymbol{n}_{N}, l\right)}{m}
$$

where $l$ denotes a label and $r$ is the result of Von Neumann's projective measurement of the dichotomic observables parameterized by the directions of $\boldsymbol{n}_{1}, \boldsymbol{n}_{2}, \cdots, \boldsymbol{n}_{N}$. 
Assume the quantum correlation function with the system in a pure uncorrelated state given in (6.2) admits projective measurement theory. One has the following proposition concerning projective measurement theory

$$
E_{\mathrm{QM}}\left(\boldsymbol{n}_{1}, \boldsymbol{n}_{2}, \cdots, \boldsymbol{n}_{N}\right)=\lim _{m \rightarrow \infty} \frac{\sum_{l=1}^{m} r\left(\boldsymbol{n}_{1}, \boldsymbol{n}_{2}, \cdots, \boldsymbol{n}_{N}, l\right)}{m} .
$$

In what follows, we show that we cannot assign the truth value " 1 " for the proposition (6.13) concerning projective measurement theory.

Assume the proposition (80) is true. By changing the label $l$ into $l^{\prime}$ and by changing the label $m$ into $m^{\prime}$, we have the same quantum expected value as follows

$$
E_{\mathrm{QM}}\left(\boldsymbol{n}_{1}, \boldsymbol{n}_{2}, \cdots, \boldsymbol{n}_{N}\right)=\lim _{m^{\prime} \rightarrow \infty} \frac{\sum_{l^{\prime}=1}^{m^{\prime}} r\left(\boldsymbol{n}_{1}, \boldsymbol{n}_{2}, \cdots, \boldsymbol{n}_{N}, l^{\prime}\right)}{m^{\prime}} .
$$

An important note here is that the value of the right-hand-side of (6.13) is equal to the value of the right-handside of (6.14) because we only change labels.

We abbreviate $r\left(\boldsymbol{n}_{1}, \boldsymbol{n}_{2}, \ldots, \boldsymbol{n}_{N}, l\right)$ to $r(l)$ and $r\left(\boldsymbol{n}_{1}, \boldsymbol{n}_{2}, \cdots, \boldsymbol{n}_{N}, l^{\prime}\right)$ to $r\left(l^{\prime}\right)$.

We have

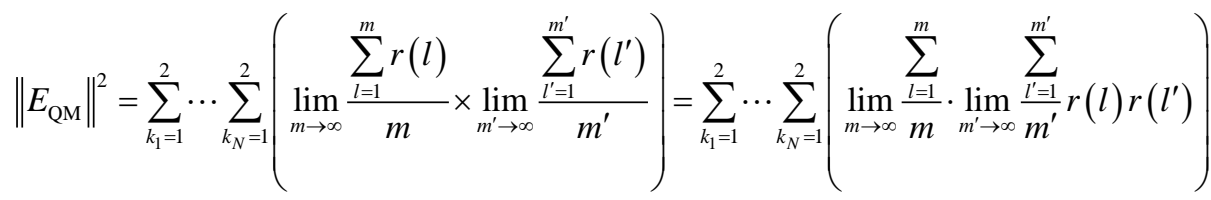

$$
\begin{aligned}
& \leq \sum_{k_{1}=1}^{2} \cdots \sum_{k_{N}=1}^{2}\left(\lim _{m \rightarrow \infty} \frac{\sum_{l=1}^{m}}{m} \cdot \lim _{m^{\prime} \rightarrow \infty} \frac{\sum_{l^{\prime}=1}^{m^{\prime}}}{m^{\prime}}\left|r(l) r\left(l^{\prime}\right)\right|\right)=\sum_{k_{1}=1}^{2} \cdots \sum_{k_{N}=1}^{2}\left(\lim _{m \rightarrow \infty} \frac{\sum_{l=1}^{m}}{m} \cdot \lim _{m^{\prime} \rightarrow \infty} \frac{\sum_{l^{\prime}=1}^{m^{\prime}}}{m^{\prime}}\right)=2^{N} .
\end{aligned}
$$

We use the following fact

$$
\left|r\left(\boldsymbol{n}_{1}, \boldsymbol{n}_{2}, \cdots, \boldsymbol{n}_{N}, l\right) r\left(\boldsymbol{n}_{1}, \boldsymbol{n}_{2}, \cdots, \boldsymbol{n}_{N}, l^{\prime}\right)\right|=+1 .
$$

The inequality (6.15) is saturated since we have

$$
\begin{aligned}
& \left\{l \mid r\left(\boldsymbol{n}_{1}, \boldsymbol{n}_{2}, \cdots, \boldsymbol{n}_{N}, l\right)=1 \wedge l \in N\right\}=\left\{l^{\prime} \mid r\left(\boldsymbol{n}_{1}, \boldsymbol{n}_{2}, \cdots, \boldsymbol{n}_{N}, l^{\prime}\right)=1 \wedge l^{\prime} \in N\right\}, \\
& \left\{l \mid r\left(\boldsymbol{n}_{1}, \boldsymbol{n}_{2}, \cdots, \boldsymbol{n}_{N}, l\right)=-1 \wedge l \in N\right\}=\left\{l^{\prime} \mid r\left(\boldsymbol{n}_{1}, \boldsymbol{n}_{2}, \cdots, \boldsymbol{n}_{N}, l^{\prime}\right)=-1 \wedge l^{\prime} \in N\right\} .
\end{aligned}
$$

Hence one has the following proposition concerning projective measurement theory

$$
\left\|E_{\mathrm{QM}}\right\|_{\max }^{2}=2^{N} .
$$

Clearly, we cannot assign the truth value " 1 " for two propositions (6.11) (concerning our physical world) and (6.18) (concerning projective measurement theory), simultaneously, when the system is in a multiparticle pure uncorrelated state. Of course, each of them is a spin-1/2 pure state lying in the $x-y$ plane. Therefore, we are in the contradiction when the system is in such a multiparticle pure uncorrelated state. Thus, we cannot accept the validity of the proposition (6.13) (concerning projective measurement theory) if we assign the truth value " 1 " for the proposition (6.11) (concerning our physical world). In other words, such projective measurement theory does not reveal our physical world.

\section{Solution of the Problem of Von Neumann's Theory}

In this section, we solve the contradiction presented in the previous section. We have the maximal possible value of the scalar product as a quantum proposition concerning our physical world 


$$
\left\|E_{\mathrm{QM}}\right\|_{\max }^{2}=1
$$

when the system is in such a multi spin-1/2 pure uncorrelated state. On the other hand, one has the following proposition concerning projective measurement theory

$$
\left\|E_{\mathrm{QM}}\right\|_{\max }^{2}=2^{N} .
$$

We cannot assign the truth value " 1 " for two propositions (7.1) (concerning our physical world) and (7.3) (concerning projective measurement theory), simultaneously, when the system is in a multiparticle pure uncorrelated state. Of course, each of them is a spin-1/2 pure state lying in the $x-y$ plane. Therefore, we are in the contradiction when the system is in such a multiparticle pure uncorrelated state.

We introduce the following hypothesis:

Hypothesis: We assume the value of $r$ is $\pm \frac{1}{\sqrt{2^{N}}}$ (in $(\hbar / 2)^{N}$ unit), which is obtained if the measurement directions are set at $\boldsymbol{n}_{1}, \boldsymbol{n}_{2}, \cdots, \boldsymbol{n}_{N}$.

When we accept this hypothesis, the proposition (7.2) (concerning projective measurement theory) becomes the following new proposition concerning a quantum measurement theory (two-setting model)

$$
\left\|E_{\mathrm{QM}}\right\|_{\max }^{2}=1 \text {. }
$$

We can assign the truth value "1" for both two propositions (7.1) (concerning our physical world) and (7.3) (concerning the quantum measurement theory), simultaneously, when the system is in a multiparticle pure uncorrelated state. Of course, each of them is a spin-1/2 pure state lying in the $x-y$ plane. Therefore, we are not in the contradiction when the system is in such a multiparticle pure uncorrelated state. Hence, we solve the contradiction presented in the previous section by changing the value of the result of quantum measurements. Our solution is equivalent to changing Planck's constant $(\hbar)$ to the new constant $(\hbar / \sqrt{2})$.

\section{New Type of the Deutsch-Jozsa Algorithm}

The earliest quantum algorithm, the Deutsch-Jozsa algorithm, is representative to show that quantum computation is faster than classical counterpart with a magnitude that grows exponentially with the number of qubits.

Let us follow the argumentation presented in [10].- The application, known as Deutsch's problem, may be described as the following game. Alice, in Amsterdam, selects a number $x$ from 0 to $2^{N}-1$, and mails it in a letter to Bob, in Boston. Bob calculates the value of some function

$$
f:\left\{0, \cdots, 2^{N}-1\right\} \rightarrow\{0,1\}
$$

and replies with the result, which is either 0 or 1 . Now, Bob has promised to use a function $f$ which is of one of two kinds; either the value of $f(x)$ is constant for all values of $x$, or else the value of $f(x)$ is balanced, that is, equal to 1 for exactly half of all the possible $x$, and 0 for the other half. Alice's goal is to determine with certainty whether Bob has chosen a constant or a balanced function, corresponding with him as little as possible. How fast can she succeed?

In the classical case, Alice may only send Bob one value of $x$ in each letter. At worst, Alice will need to query Bob at least

$$
2^{N} / 2+1
$$

times, since she may receive $2^{N} / 20 \mathrm{~s}$ before finally getting a 1 , telling her that Bob's function is balanced. The best deterministic classical algorithm she can use therefore requires $2^{N} / 2+1$ queries. Note that in each letter, Alice sends Bob $N$ bits of information. Furthermore, in this example, physical distance is being used to artificially elevate the cost of calculating $f(x)$, but this is not needed in the general problem, where $f(x)$ may be inherently difficult to calculate.

If Bob and Alice were able to exchange qubits, instead of just classical bits, and if Bob agreed to calculate $f(x)$ using a unitary transformation $U_{f}$, then Alice could achieve her goal in just one correspondence with 
Bob, using the following algorithm.

Alice has an $N$ qubit register to store her query in, and a single qubit register which she will give to Bob, to store the answer in. She begins by preparing both her query and answer registers in a superposition state. Bob will evaluate $f(x)$ using quantum parallelism and leave the result in the answer register. Alice then interferes states in the superposition using a Hadamard transformation (a unitary transformation),

$$
H=\left(\sigma_{x}+\sigma_{z}\right) / \sqrt{2},
$$

on the query register, and finishes by performing a suitable measurement to determine whether $f$ was constant or balanced.

Let us follow the quantum states through this algorithm. The input state is

$$
\left|\psi_{0}\right\rangle=|0\rangle^{\otimes N}|1\rangle \text {. }
$$

Here the query register describes the state of $N$ qubits all prepared in the

state. After the Hadamard transformation on the query register and the Hadamard gate on the answer register we have

$$
\left|\psi_{1}\right\rangle=\sum_{x \in\{0,1\}^{N}} \frac{|x\rangle}{\sqrt{2^{N}}}\left[\frac{|0\rangle-|1\rangle}{\sqrt{2}}\right] .
$$

The query register is now a superposition of all values, and the answer register is in an evenly weighted superposition of

and

$$
|1\rangle \text {. }
$$

Next, the function $f$ is evaluated (by Bob) using

$$
U_{f}:|x, y\rangle \rightarrow|x, y \oplus f(x)\rangle,
$$

giving

$$
\left|\psi_{2}\right\rangle= \pm \sum_{x} \frac{(-1)^{f(x)}|x\rangle}{\sqrt{2^{N}}}\left[\frac{|0\rangle-|1\rangle}{\sqrt{2}}\right] .
$$

Here

$$
y \oplus f(x)
$$

is the bitwise XOR (exclusive OR) of $y$ and $f(x)$. Alice now has a set of qubits in which the result of Bob's function evaluation is stored in the amplitude of the qubit superposition state. She now interferes terms in the superposition using a Hadamard transformation on the query register. To determine the result of the Hadamard transformation it helps to first calculate the effect of the Hadamard transformation on a state

$$
|x\rangle \text {. }
$$

By checking the cases $x=0$ and $x=1$ separately we see that for a single qubit

$$
H|x\rangle=\sum_{z}(-1)^{x z}|z\rangle / \sqrt{2} .
$$

Thus 


$$
H^{\otimes N}\left|x_{1}, \cdots, x_{N}\right\rangle=\frac{\sum_{z_{1}, \cdots, z_{N}}(-1)^{{x_{1} z_{1}+\cdots+x_{N} z_{N}}}\left|z_{1}, \cdots, z_{N}\right\rangle}{\sqrt{2^{N}}} .
$$

This can be summarized more succinctly in the very useful equation

$$
H^{\otimes N}|x\rangle=\frac{\sum_{z}(-1)^{x \cdot z}|z\rangle}{\sqrt{2^{N}}},
$$

where

$$
x \cdot z
$$

is the bitwise inner product of $x$ and $z$, modulo 2. Using this equation and (8.10) we can now evaluate $\left|\psi_{3}\right\rangle$,

$$
\left|\psi_{3}\right\rangle= \pm \sum_{z} \sum_{x} \frac{(-1)^{x \cdot z+f(x)}|z\rangle}{\sqrt{2^{N}}}\left[\frac{|0\rangle-|1\rangle}{\sqrt{2}}\right] .
$$

Alice now observes the query register. Note that the absolute value of the amplitude for the state

$$
|0\rangle^{\otimes N}
$$

is

$$
\sum_{x}(-1)^{f(x)} / 2^{N}
$$

Let's look at the two possible cases- $f$ constant and $f$ balanced-to discern what happens. In the case where $f$ is constant the absolute value of the amplitude for

$$
|0\rangle^{\otimes N}
$$

is +1 . Because

$$
\left|\psi_{3}\right\rangle
$$

is of unit length it follows that all the other amplitudes must be zero, and an observation will yield

$$
\left(+\frac{1}{\sqrt{2}}\right)
$$

times for all $N$ qubits in the query register. Thus, global measurement outcome is

$$
\left(+\frac{1}{\sqrt{2^{N}}}\right)
$$

If $f$ is balanced then the positive and negative contributions to the absolute value of the amplitude for

$$
|0\rangle^{\otimes N}
$$

cancel, leaving an amplitude of zero, and a measurement must yield a result other than

$$
+\frac{1}{\sqrt{2}} \text {, }
$$

that is,

$$
-\frac{1}{\sqrt{2}} \text {, }
$$


on at least one qubit in the query register. Summarizing, if Alice measures all $\left(+\frac{1}{\sqrt{2}}\right) \mathrm{s}$ and global measurement outcome is $\left(+\frac{1}{\sqrt{2^{N}}}\right)$ the function is constant; otherwise the function is balanced.

We notice that the difference between $+\frac{1}{\sqrt{2^{N}}}$ and $-\frac{1}{\sqrt{2^{N}}}$ is approximately zero when $N \gg 1$. We question if the Deutsch-Jozsa algorithm in the macroscopic scale is possible or not. This question is open problem.

\section{Double-Slit Experiment and Projective Measurement Theory}

In this section, we consider the relation between double-slit experiment and projective measurement theory. We try to implement double-slit experiment. There is a detector just after each slit. Thus interference figure does not appear, and we do not consider such a pattern. The possible values of the result of measurements are \pm 1 (in $\hbar / 2$ unit). If a particle passes one side slit, then the value of the result of measurement is +1 . If a particle passes through another slit, then the value of the result of measurement is -1 .

\subsection{A Wave Function Analysis}

Let $\left(\sigma_{z}, \sigma_{x}\right)$ be Pauli vector. We assume that a source of spin-carrying particles emits them in a state $|\psi\rangle$, which can be described as an eigenvector of Pauli observable $\sigma_{z}$. We consider a quantum expected value $\left\langle\sigma_{x}\right\rangle$ as

$$
\left\langle\sigma_{x}\right\rangle=\left\langle\psi\left|\sigma_{x}\right| \psi\right\rangle=0
$$

The above quantum expected value is zero if we consider only a wave function analysis.

We derive a necessary condition for the quantum expected value for the system in the pure spin-1/2 state $|\psi\rangle$ given in (115). We derive the possible value of the product $\left\langle\sigma_{x}\right\rangle \times\left\langle\sigma_{x}\right\rangle=\left\langle\sigma_{x}\right\rangle^{2} .\left\langle\sigma_{x}\right\rangle$ is the quantum expected value given in (115). We derive the following proposition

$$
\left\langle\sigma_{x}\right\rangle^{2}=0
$$

Hence we have

$$
\left\langle\sigma_{x}\right\rangle^{2} \leq 0
$$

Thus,

$$
\left(\left\langle\sigma_{x}\right\rangle^{2}\right)_{\max }=0
$$

\subsection{Projective Measurement Theory}

On the other hand, a mean value $E$ admits projective measurement theory if it can be written as

$$
E=\frac{\sum_{l=1}^{m} r_{l}\left(\sigma_{x}\right)}{m}
$$

where $l$ denotes a label and $r$ is the result of projective measurement of the Pauli observable $\sigma_{x}$. We assume the value of $r$ is \pm 1 (in $\hbar / 2$ unit).

Assume the quantum mean value with the system in an eigenvector $(|\psi\rangle)$ of Pauli observable $\sigma_{z}$ given in (9.1) admits projective measurement theory. One has the following proposition concerning projective measurement theory

$$
\left\langle\sigma_{x}\right\rangle(m)=\frac{\sum_{l=1}^{m} r_{l}\left(\sigma_{x}\right)}{m}
$$


We can assume as follows by Strong Law of Large Numbers,

$$
\left\langle\sigma_{x}\right\rangle(+\infty)=\left\langle\sigma_{x}\right\rangle=\left\langle\psi\left|\sigma_{x}\right| \psi\right\rangle .
$$

In what follows, we show that we cannot assign the truth value " 1 " for the proposition (9.6) concerning projective measurement theory.

Assume the proposition (9.6) is true. By changing the label $l$ into $l^{\prime}$ and by changing the label $m$ into $m^{\prime}$, we have the same quantum mean value as follows

$$
\left\langle\sigma_{x}\right\rangle\left(m^{\prime}\right)=\frac{\sum_{I^{\prime}=1}^{m^{\prime}} r_{l^{\prime}}\left(\sigma_{x}\right)}{m^{\prime}} .
$$

An important note here is that the value of the right-hand-side of (9.6) is equal to the value of the right-hand-side of (9.8) because we only change labels. We have

$$
\begin{aligned}
\left\langle\sigma_{x}\right\rangle(m) \times\left\langle\sigma_{x}\right\rangle\left(m^{\prime}\right)= & \frac{\sum_{l=1}^{m} r_{l}\left(\sigma_{x}\right)}{m} \times \frac{\sum_{l^{\prime}=1}^{m^{\prime}} r_{l^{\prime}}\left(\sigma_{x}\right)}{m^{\prime}}=\frac{\sum_{l=1}^{m}}{m} \cdot \frac{\sum_{l^{\prime}=1}^{m^{\prime}}}{m^{\prime}} r_{l}\left(\sigma_{x}\right) r_{l^{\prime}}\left(\sigma_{x}\right) \\
& \leq \frac{\sum_{l=1}^{m}}{m} \cdot \frac{\sum_{l^{\prime}=1}^{m^{\prime}}}{m^{\prime}}\left|r_{l}\left(\sigma_{x}\right) r_{l^{\prime}}\left(\sigma_{x}\right)\right|=\frac{\sum_{l=1}^{m}}{m} \cdot \frac{\sum_{l^{\prime}=1}^{m^{\prime}}}{m^{\prime}}=1 .
\end{aligned}
$$

We use the following fact

$$
\left|r_{l}\left(\sigma_{x}\right) r_{l^{\prime}}\left(\sigma_{x}\right)\right|=1
$$

The inequality (9.9) is saturated since we have

$$
\begin{aligned}
& \left\{l \mid r_{l}\left(\sigma_{x}\right)=1 \wedge l \in \mathbf{N}\right\}=\left\{l^{\prime} \mid r_{l^{\prime}}\left(\sigma_{x}\right)=1 \wedge l^{\prime} \in \mathbf{N}\right\}, \\
& \left\{l \mid r_{l}\left(\sigma_{x}\right)=-1 \wedge l \in \mathbf{N}\right\}=\left\{l^{\prime} \mid r_{l^{\prime}}\left(\sigma_{x}\right)=-1 \wedge l^{\prime} \in \mathbf{N}\right\} .
\end{aligned}
$$

Thus we derive a proposition concerning the quantum mean value under an assumption that projective measurement theory is true (in a spin-1/2 system), that is

$$
\left(\left\langle\sigma_{x}\right\rangle(m) \times\left\langle\sigma_{x}\right\rangle\left(m^{\prime}\right)\right)_{\max }=1 .
$$

From Strong Law of Large Numbers, we have

$$
\left(\left\langle\sigma_{x}\right\rangle \times\left\langle\sigma_{x}\right\rangle\right)_{\max }=1 .
$$

Hence we derive the following proposition concerning projective measurement theory

$$
\left(\left\langle\sigma_{x}\right\rangle^{2}\right)_{\max }=1 \text {. }
$$

We do not assign the truth value " 1 " for two propositions (9.4) (concerning a wave function analysis) and (9.14) (concerning projective measurement theory), simultaneously. We are in the contradiction. This implies that we cannot perform the following Deutsch's algorithm.

- The control of quantum states relies on the wave functional analysis.

- The observation of quantum states relies on projective measurement theory.

We cannot accept the validity of the proposition (9.6) (concerning projective measurement theory) if we assign the truth value "1" for the proposition (9.4) (concerning a wave function analysis). In other words, such projective measurement theory does not meet the detector model for spin observable $\sigma_{x}$. And we cannot perform Deutsch's algorithm. Consistency between controlability and observability is necessary for an implementation of Deutsch's algorithm. And desired consistency is not established. 


\section{Solution of the Problem of Projective Measurement Theory in a Macroscopic System}

In this section, we consider many double-slit experiments. In a macroscopic system, we solve the contradiction presented in the previous section.

\subsection{A Wave Function analysis}

We consider an implementation of $N$ double-slit experiments. We assume that $N$ sources of spin-carrying particles emit them in a state, which can be described as an eigenvector of Pauli observable $\sigma_{z}$. We have the following state globally

$$
\overbrace{|\psi\rangle|\psi\rangle \cdots|\psi\rangle}^{N}=|\psi\rangle^{\otimes N}
$$

Each of them can be described as an eigenvector of Pauli observable $\sigma_{z}$. We analyze experimental data globally. We consider a single expected value of

$$
\overbrace{\sigma_{x} \otimes \sigma_{x} \otimes \cdots \otimes \sigma_{x}}^{N}=\sigma_{x}^{\otimes N}
$$

then we have the following quantum expected value from a wave function analysis

$$
\left\langle\left.\psi\right|^{\otimes N} \sigma_{x}^{\otimes N} \mid \psi\right\rangle^{\otimes N}=\left(\left\langle\psi\left|\sigma_{x}\right| \psi\right\rangle\right)^{N}=\left\langle\sigma_{x}\right\rangle^{N}=0
$$

Thus we have the following proposition concerning a wave function analysis

$$
\left(\left\langle\sigma_{x}\right\rangle^{2}\right)^{N}=0,(N \rightarrow+\infty)
$$

Hence we have

$$
\left(\left\langle\sigma_{x}\right\rangle\right)^{2 N} \leq 0,(N \rightarrow+\infty)
$$

Thus,

$$
\left(\left\langle\sigma_{x}\right\rangle^{2}\right)_{\max }^{N}=0,(N \rightarrow+\infty)
$$

\subsection{New Type of a Quantum Measurement}

On the other hand, a mean value $E$ admits a quantum measurement theory if it can be written as

$$
E=\frac{\sum_{l=1}^{m} r_{l}\left(\sigma_{x}\right)}{m}
$$

where $l$ denotes a label and $r$ is the result of quantum measurement of the Pauli observable $\sigma_{x}$. We assume the value of $r$ is $\pm \frac{1}{\sqrt{2}}$ (in $\hbar / 2$ unit) [23]. If a particle passes one side slit, then the value of the result of measurement is $+\frac{1}{\sqrt{2}}$. If a particle passes through another slit, then the value of the result of measurement is $-\frac{1}{\sqrt{2}}$

Assume the quantum mean value with the system in an eigenvector $(|\psi\rangle)$ of the Pauli observable $\sigma_{z}$ given in (9.1) admits such a quantum measurement theory. One has the following proposition concerning the quantum measurement theory 


$$
\left\langle\sigma_{x}\right\rangle(m)=\frac{\sum_{l=1}^{m} r_{l}\left(\sigma_{x}\right)}{m} .
$$

In what follows, we show that we can assign the truth value "1" for the proposition (10.8) concerning the quantum measurement theory in the macroscopic system $(N \rightarrow+\infty)$.

Assume the proposition (10.8) is true. By changing the label $l$ into $l^{\prime}$ and by changing the label $m$ into $m^{\prime}$, we have the same quantum mean value as follows

$$
\left\langle\sigma_{x}\right\rangle\left(m^{\prime}\right)=\frac{\sum_{l^{\prime}=1}^{m^{\prime}} r_{l^{\prime}}\left(\sigma_{x}\right)}{m^{\prime}} .
$$

An important note here is that the value of the right-hand-side of (10.8) is equal to the value of the right-handside of (10.9) because we only change labels. We have

$$
\begin{aligned}
\left\langle\sigma_{x}\right\rangle(m) \times\left\langle\sigma_{x}\right\rangle\left(m^{\prime}\right) & =\frac{\sum_{l=1}^{m} r_{l}\left(\sigma_{x}\right)}{m} \times \frac{\sum_{l^{\prime}=1}^{m^{\prime}} r_{l^{\prime}}\left(\sigma_{x}\right)}{m^{\prime}}=\frac{\sum_{l=1}^{m}}{m} \cdot \frac{\sum_{l^{\prime}=1}^{m^{\prime}}}{m^{\prime}} r_{l}\left(\sigma_{x}\right) r_{l^{\prime}}\left(\sigma_{x}\right) \\
& \leq \frac{\sum_{l=1}^{m}}{m} \cdot \frac{\sum_{l^{\prime}=1}^{m^{\prime}}}{m^{\prime}}\left|r_{l}\left(\sigma_{x}\right) r_{l^{\prime}}\left(\sigma_{x}\right)\right|=(1 / 2) \frac{\sum_{l=1}^{m}}{m} \cdot \frac{\sum_{l^{\prime}=1}^{m^{\prime}}}{m^{\prime}}=1 / 2 .
\end{aligned}
$$

We use the following fact

$$
\left|r_{l}\left(\sigma_{x}\right) r_{l^{\prime}}\left(\sigma_{x}\right)\right|=1 / 2
$$

The inequality (10.10) is saturated since we have

$$
\begin{aligned}
& \left\{l \mid r_{l}\left(\sigma_{x}\right)=1 / \sqrt{2} \wedge l \in \mathbf{N}\right\}=\left\{l^{\prime} \mid r_{l^{\prime}}\left(\sigma_{x}\right)=1 / \sqrt{2} \wedge l^{\prime} \in \mathbf{N}\right\}, \\
& \left\{l \mid r_{l}\left(\sigma_{x}\right)=-1 / \sqrt{2} \wedge l \in \mathbf{N}\right\}=\left\{l^{\prime} \mid r_{l^{\prime}}\left(\sigma_{x}\right)=-1 / \sqrt{2} \wedge l^{\prime} \in \mathbf{N}\right\} .
\end{aligned}
$$

Thus we derive a proposition concerning the quantum mean value under an assumption that such a quantum measurement is true (in a spin-1/2 system), that is,

$$
\left(\left\langle\sigma_{x}\right\rangle(m) \times\left\langle\sigma_{x}\right\rangle\left(m^{\prime}\right)\right)_{\max }=1 / 2 .
$$

From Strong Law of Large Numbers, we have

$$
\left(\left\langle\sigma_{x}\right\rangle \times\left\langle\sigma_{x}\right\rangle\right)_{\max }=1 / 2
$$
Therefore we have $\left(\left\langle\sigma_{x}\right\rangle^{2}\right)_{\max }^{N}=1 / 2^{N}$. Hence we derive the following proposition concerning the quantum
measurement

$$
\left(\left\langle\sigma_{x}\right\rangle^{2}\right)_{\max }^{N}=1 / 2^{N}
$$

Thus,

$$
\left(\left\langle\sigma_{x}\right\rangle^{2}\right)_{\max }^{N}=0,(N \rightarrow+\infty) .
$$

We can assign the truth value "1" for both two propositions (10.6) (concerning a wave function analysis) and (10.16) (concerning the quantum measurement theory), simultaneously. Hence, we solve the contradiction presented in the previous section by changing the value of the result of quantum measurements and by considering an implementation of double-slit experiments macroscopically. This implies that we can perform the following Deutsch's algorithm. 
- The control of quantum states relies on the wave functional analysis.

- The observation of quantum states relies on the measurement theory.

In other words, such a measurement theory meets the detector model for spin observable $\sigma_{x}$. And we can perform Deutsch's algorithm. Consistency between controlability and observability is necessary for an implementation of Deutsch's algorithm. And desired consistency is established.

\section{The Relation between Our Result and Deutsch's Algorithm}

In this section, we discuss how our solution is used in an implementation of Deutsch's algorithm. Now, we can measure Pauli observable $\sigma_{x}$ by solving the contradiction discussed in Section 10. Consistency between controlability and observability is established. The values of the result of quantum measurements are $\pm 1 / \sqrt{2}$. So the values can be used for the values of the result of the final measurement of Deutsch's algorithm. From Section 4 , we have

$$
\left|\psi_{2}\right\rangle= \begin{cases} \pm\left[\frac{|0\rangle+|1\rangle}{\sqrt{2}}\right]\left[\frac{|0\rangle-|1\rangle}{\sqrt{2}}\right] & \text { if } f(0)=f(1) \\ \pm\left[\frac{|0\rangle-|1\rangle}{\sqrt{2}}\right]\left[\frac{|0\rangle-|1\rangle}{\sqrt{2}}\right] & \text { if } f(0) \neq f(1)\end{cases}
$$

We can consider

$$
\pm\left[\frac{|0\rangle+|1\rangle}{\sqrt{2}}\right]= \pm\left|+{ }_{x}\right\rangle, \pm\left[\frac{|0\rangle-|1\rangle}{\sqrt{2}}\right]= \pm\left|-{ }_{x}\right\rangle .
$$

Therefore if we can measure an expected value of $\sigma_{x}$, then we can distinguish the two states mentioned above. From a wave function analysis, we have

$$
\left\langle+_{x}\left|\sigma_{x}\right|+_{x}\right\rangle=+1,\left\langle-_{x}\left|\sigma_{x}\right|-{ }_{x}\right\rangle=-1 .
$$

We see one measurement is enough to determine which state is realized. We can omit the final Hadamard gate on the first qubit.

\section{Conclusions}

In conclusion, we have discussed the fact that there is a crucial contradiction within Von Neumann's theory. We have derived a proposition concerning a quantum expected value under an assumption of the existence of the orientation of reference frames in $N$ spin-1/2 systems $(1 \leq N<+\infty)$. This assumption intuitively has depictured our physical world. However, the quantum predictions within the formalism of Von Neumann's projective measurement have violated the proposition with a magnitude that grows exponentially with the number of particles. We have had to give up either the existence of the directions or the formalism of Von Neumann's projective measurement. Therefore, Von Neumann's theory cannot have depictured our physical world with a violation factor that grows exponentially with the number of particles. The theoretical formalism of the implementation of the Deutsch-Jozsa algorithm has relied on Von Neumann's theory. We have investigated whether Von Neumann's theory meets the Deutsch-Jozsa algorithm. We have discussed the fact that the crucial contradiction makes the quantum-theoretical formulation of Deutsch-Jozsa algorithm questionable. Further, we have discussed the fact that projective measurement theory does not meet easy detector model for a single Pauli observable. Especially, we have systematically described our assertion based on more mathematical analysis using raw data. We have proposed a solution of the problem. Our solution has been equivalent to changing Planck's constant $(\hbar)$ to the new constant $(\hbar / \sqrt{2})$. It may have said that a new type of the quantum theory early approaches Newton's theory in the macroscopic scale than the old quantum theory does. We have discussed how our solution is used in an implementation of Deutsch's algorithm.

What are new physical theories? We cannot answer it at this stage. However, we expect that our discussion in this paper could contribute to creating new physical theories in order to explain our physical world, to create new information science, and to predict new unknown physical phenomena efficiently. 


\section{Acknowledgements}

We would like to thank Professor Niizeki and Dr. Ren for valuable comments.

\section{References}

[1] von Neumann, J. (1955) Mathematical Foundations of Quantum Mechanics. Princeton University Press, Princeton.

[2] Nagata, K., Ren, C.-L. and Nakamura, T. (2011) Whether Quantum Computation Can Be Almighty. Advanced Studies in Theoretical Physics, 5, 1-14.

[3] Gershenfeld, N. and Chuang, I.L. (1998) Quantum Computing with Molecules. Scientific American.

[4] Deutsch, D. (1992) Quantum Computation. Physics World, 1/6/92.

[5] Quantum Computer. Wikipedia, the Free Encyclopedia.

[6] Feynman, R.P., Leighton, R.B. and Sands, M. (1965) Lectures on Physics, Volume III, Quantum Mechanics. Addison-Wesley Publishing Company, Reading.

[7] Redhead, M. (1989) Incompleteness, Nonlocality, and Realism. 2nd Edition, Clarendon Press, Oxford.

[8] Peres, A. (1993) Quantum Theory: Concepts and Methods. Kluwer Academic, Dordrecht.

[9] Sakurai, J.J. (1995) Modern Quantum Mechanics. Revised Edition, Addison-Wesley Publishing Company, Reading.

[10] Nielsen, M.A. and Chuang, I.L. (2000) Quantum Computation and Quantum Information. Cambridge University Press, Cambridge.

[11] Leggett, A.J. (2003) Nonlocal Hidden-Variable Theories and Quantum Mechanics: An Incompatibility Theorem. Foundations of Physics, 33, 1469-1493. http://dx.doi.org/10.1023/A:1026096313729

[12] Gröblacher, S., Paterek, T., Kaltenbaek, R., Brukner, Č., Żukowski, M., Aspelmeyer, M. and Zeilinger, A. (2007) An Experimental Test of Non-Local Realism. Nature, 446, 871-875. http://dx.doi.org/10.1038/nature05677

[13] Paterek, T., Fedrizzi, A., Gröblacher, S., Jennewein, T., Żukowski, M., Aspelmeyer, M. and Zeilinger, A. (2007) Experimental Test of Nonlocal Realistic Theories without the Rotational Symmetry Assumption. Physical Review Letters, 99, Article ID: 210406. http://dx.doi.org/10.1103/PhysRevLett.99.210406

[14] Branciard, C., Ling, A., Gisin, N., Kurtsiefer, C., Lamas-Linares, A. and Scarani, V. (2007) Experimental Falsification of Leggett’s Nonlocal Variable Model. Physical Review Letters, 99, Article ID: 210407. http://dx.doi.org/10.1103/PhysRevLett.99.210407

[15] Deutsch, D. (1985) Quantum Theory, the Church-Turing Principle and the Universal Quantum Computer. Proceedings of the Royal Society A: Mathematical, Physical and Engineering Sciences, 400, 97-117. http://dx.doi.org/10.1098/rspa.1985.0070

[16] Jones, J.A. and Mosca, M. (1998) Implementation of a Quantum Algorithm on a Nuclear Magnetic Resonance Quantum Computer. The Journal of Chemical Physics, 109, 1648. http://dx.doi.org/10.1063/1.476739

[17] Gulde, S., Riebe, M., Lancaster, G.P.T., Becher, C., Eschner, J., Häffner, H., Schmidt-Kaler, F., Chuang, I.L. and Blatt, R. (2003) Implementation of the Deutsch-Jozsa Algorithm on an Ion-Trap Quantum Computer. Nature, 421, 48-50. http://dx.doi.org/10.1038/nature01336

[18] de Oliveira, A.N., Walborn, S.P. and Monken, C.H. (2005) Implementing the Deutsch Algorithm with Polarization and Transverse Spatial Modes. Journal of Optics B: Quantum and Semiclassical Optics, 7, 288-292. http://dx.doi.org/10.1088/1464-4266/7/9/009

[19] Kim, Y.-H. (2003) Single-Photon Two-Qubit Entangled States: Preparation and Measurement. Physical Review A, 67, Article ID: 040301(R).

[20] Mohseni, M., Lundeen, J.S., Resch, K.J. and Steinberg, A.M. (2003) Experimental Application of Decoherence-Free Subspaces in an Optical Quantum-Computing Algorithm. Physical Review Letters, 91, Article ID: 187903. http://dx.doi.org/10.1103/PhysRevLett.91.187903

[21] Tame, M.S., Prevedel, R., Paternostro, M., Böhi, P., Kim, M.S. and Zeilinger, A. (2007) Experimental Realization of Deutsch’s Algorithm in a One-Way Quantum Computer. Physical Review Letters, 98, Article ID: 140501. http://dx.doi.org/10.1103/PhysRevLett.98.140501

[22] Deutsch, D. and Jozsa, R. (1992) Rapid Solution of Problems by Quantum Computation. Proceedings of the Royal Society A, 439, 553-558. http://dx.doi.org/10.1098/rspa.1992.0167

[23] Nagata, K. and Nakamura, T. (2010) Can von Neumann’s Theory Meet the Deutsch-Jozsa Algorithm? International Journal of Theoretical Physics, 49, 162-170. http://dx.doi.org/10.1007/s10773-009-0189-5

[24] Nagata, K. (2009) There Is No Axiomatic System for the Quantum Theory. International Journal of Theoretical Physics, 48, 3532-3536. http://dx.doi.org/10.1007/s10773-009-0158-z 
[25] Nagata, K. and Nakamura, T. (2013) Von Neumann’s Theory Does Not Meet Deutsch’s Algorithm. Precision Instrument and Mechanology, 2, 104.

[26] Nagata, K. and Nakamura, T. (2013) An Additional Condition for Bell Experiments for Accepting Local Realistic Theories. Quantum Information Processing, 12, 3785-3789. http://dx.doi.org/10.1007/s11128-013-0635-4

[27] Nagata, K. (2014) Reply to "Comments on 'There Is No Axiomatic System for the Quantum Theory”". Journal of Quantum Information Science, 4, 195-200. http://dx.doi.org/10.4236/jqis.2014.44018

[28] De Broglie-Bohm Theory-Wikipedia, the Free Encyclopedia.

[29] Schon, C. and Beige, A. (2001) Analysis of a Two-Atom Double-Slit Experiment Based on Environment-Induced Measurements. Physical Review A, 64, Article ID: 023806. http://dx.doi.org/10.1103/PhysRevA.64.023806 